Article

\title{
New Refinement of the Operator Kantorovich Inequality
}

\author{
Hamid Reza Moradi ${ }^{1, * \mathbb{D}}$, Shigeru Furuichi ${ }^{2} \mathbb{D}$ and Zahra Heydarbeygi ${ }^{1}$ \\ 1 Department of Mathematics, Payame Noor University (PNU), P.O. Box 19395-4697, Tehran, Iran; \\ zheydarbeygi@yahoo.com \\ 2 Department of Information Science, College of Humanities and Sciences, Nihon University, Tokyo 102-0074, \\ Japan; furuichi@chs.nihon-u.ac.jp \\ * Correspondence: hrmoradi@mshdiau.ac.ir or hrmoradi.68@gmail.com; Tel.: +98-915-529-5869
}

Received: 7 December 2018; Accepted: 24 January 2019; Published: 1 February 2019

Abstract: We focus on the improvement of operator Kantorovich type inequalities. Among the consequences, we improve the main result of the paper [H.R. Moradi, I.H. Gümüş, Z. Heydarbeygi, A glimpse at the operator Kantorovich inequality, Linear Multilinear Algebra, doi:10.1080/ 03081087.2018.1441799].

Keywords: operator inequality; positive linear map; operator Kantorovich inequality; geometrically convex function

MSC: Primary 47A63; Secondary 46L05; 47A60

\section{Notation and Preliminaries}

At the beginning of this paper, we cite the following inequality which is called the operator Kantorovich inequality [1]:

$$
\Phi\left(A^{-1}\right) \leq \frac{(M+m)^{2}}{4 M m} \Phi(A)^{-1}
$$

where $\Phi$ is a normalized positive linear map from $\mathcal{B}(\mathcal{H})$ to $\mathcal{B}(\mathcal{K})$, (we represent $\mathcal{H}$ and $\mathcal{K}$ as complex Hilbert spaces throughout the paper) and $A$ is a positive operator with spectrum contained in $[m, M]$ with $0<m<M$. This is a non-commutative analogue of the classical inequality [2],

$$
\langle A x, x\rangle\left\langle A^{-1} x, x\right\rangle \leq \frac{(M+m)^{2}}{4 M m}
$$

where $x \in \mathcal{H}$ is a unit vector.

In recent years, various attempts have been made by many authors to improve and generalize the operator Kantorovich inequality. One may see the basic references [3-5] and the excellent survey [6] on this topic. In [7], it was shown that

$$
\Phi\left(A^{-1}\right) \leq \Phi\left(m^{\frac{A-M I}{M-m}} M^{\frac{m I-A}{M-m}}\right) \leq \frac{(M+m)^{2}}{4 M m} \Phi(A)^{-1} .
$$


The main aim of the present short paper is to improve both inequalities in (2). Actually, we prove that

$$
\begin{aligned}
\Phi\left(A^{-1}\right) & \leq \Phi\left(\left(A-(\sqrt{m}-\sqrt{M})^{2} r(A)\right)^{-1}\right) \\
& \leq \Phi\left(\left(m^{\frac{A-M I}{M-m}} M^{\frac{m I-A}{M-m}}\right)^{-1}\right) \\
& \leq \frac{(M+m)^{2}}{4 M m} \Phi(A)^{-1}-\left(\frac{(\sqrt{M}-\sqrt{m})^{2}}{M m}\right) r(A)
\end{aligned}
$$

where $r(A)=\min \left\{\frac{M I-A}{M-m}, \frac{A-m I}{M-m}\right\}=\frac{1}{2} I-\frac{1}{M-m}\left|A-\frac{M+m}{2} I\right|$.

In what follows, an operator means a bounded linear one acting on a complex Hilbert space $\mathcal{H}$. As customary, we reserve $m, M$ for scalars and $I$ for the identity operator. A self-adjoint operator $A$ is said to be positive if $\langle A x, x\rangle \geq 0$ holds for all $x \in \mathcal{H}$. A linear map $\Phi$ is positive if $\Phi(A) \geq 0$ whenever $A \geq 0$. It is said to be normalized if $\Phi(I)=I$. We denote by $\sigma(A)$ the spectrum of the operator $A$.

\section{Main Results}

Before we present the proof of our theorems, we begin with a general observation. We say that a non-negative function $f$ on $[0, \infty)$ is geometrically convex [8] when

$$
f\left(a^{1-v} b^{v}\right) \leq f(a)^{1-v} f(b)^{v}
$$

for all $a, b>0$ and $v \in[0,1]$. Equivalently, a function $f$ is geometrically convex if and only if the associated function $F(y)=\log \left(f\left(e^{y}\right)\right)$ is convex.

Example 1 ([9] Example 2.12). Given real numbers $c_{i} \geq 0$ and $p_{i} \in(-\infty, 0] \cup[1, \infty)$ for $i=1, \cdots, n$, the function $f(t)=\sum_{i=1}^{n} c_{i} t p_{i}$ is geometrically convex on $(0, \infty)$.

Kittaneh and Manasrah [10] Theorem 2.1 obtained a refinement of the weighted arithmetic-geometric mean inequality as follows:

$$
a^{1-v} b^{v} \leq(1-v) a+v b-r(\sqrt{a}-\sqrt{b})^{2}
$$

where $r=\min \{v, 1-v\}$.

Now, if $f$ is a decreasing geometrically convex function, then

$$
\begin{aligned}
f((1-v) a+v b) & \leq f\left(((1-v) a+v b)-r(\sqrt{a}-\sqrt{b})^{2}\right) \\
& \leq f\left(a^{1-v} b^{v}\right) \\
& \leq f(a)^{1-v} f(b)^{v} \\
& \leq(1-v) f(a)+v f(b)-r(\sqrt{f(a)}-\sqrt{f(b)})^{2} \\
& \leq(1-v) f(a)+v f(b)
\end{aligned}
$$


where the first inequality follows from the inequality $(1-v) a+v b-r(\sqrt{a}-\sqrt{b})^{2} \leq(1-v) a+v b$ and the fact that $f$ is decreasing function, in the second inequality we used (4), the third inequality is obvious by (3), and the fourth inequality again follows from (4) by interchanging $a$ by $f(a)$ and $b$ by $f(b)$.

Of course, each decreasing geometrically convex function is also convex. However, the converse does not hold in general.

The inequality (5) applied to $a=m, b=M, 1-v=\frac{M-t}{M-m}$, and $v=\frac{t-m}{M-m}$ gives

$$
\begin{aligned}
f(t) & \leq f\left(t-(\sqrt{m}-\sqrt{M})^{2} r(t)\right) \\
& \leq f\left(m^{\frac{M-t}{M-m}} M^{\frac{t-m}{M-m}}\right) \\
& \leq f(m)^{\frac{M-t}{M-m}} f(M)^{\frac{t-m}{M-m}} \\
& \leq \frac{M-t}{M-m} f(m)+\frac{t-m}{M-m} f(M)-(\sqrt{f(m)}-\sqrt{f(M)})^{2} r(t) \\
& \leq \frac{M-t}{M-m} f(m)+\frac{t-m}{M-m} f(M)
\end{aligned}
$$

with $r(t)=\min \left\{\frac{t-m}{M-m}, \frac{M-t}{M-m}\right\}=\frac{1}{2}-\frac{1}{M-m}\left|t-\frac{M+m}{2}\right|$ whenever $t \in[m, M]$.

In order to establish our promised refinement of the operator Kantorovich inequality, we also use the well-known monotonicity principle for bounded self-adjoint operators on Hilbert space (see, e.g., [6] (p. 3)): If $A \in \mathcal{B}(\mathcal{H})$ is a self-adjoint operator, then

$$
f(t) \leq g(t), t \in \sigma(A) \Rightarrow f(A) \leq g(A)
$$

provided that $f$ and $g$ are real-valued continuous functions. Under the same assumptions, $h(t)=|t|$ implies $h(A)=|A|$.

Now, we are in a position to state and prove our main results. We remark that the following theorem can be regarded as an extension of [5] Remark 4.14 to the context of geometrical convex functions.

Theorem 1. Let $A \in \mathcal{B}(\mathcal{H})$ be a self-adjoint operator with $\sigma(A) \subseteq[m, M]$ for some scalars $m, M$ with $0<m<M$ and $\Phi$ be a normalized positive linear map from $\mathcal{B}(\mathcal{H})$ to $\mathcal{B}(\mathcal{K})$. If $f$ is strictly positive decreasing geometrically convex function, then

$$
\begin{aligned}
\Phi\left(f\left(A-(\sqrt{m}-\sqrt{M})^{2} r(A)\right)\right) & \leq \Phi\left(f\left(m^{\frac{M I-A}{M-m}} M^{\frac{A-m I}{M-m}}\right)\right) \\
& \leq \mu(m, M, f) f(\Phi(A))-(\sqrt{f(m)}-\sqrt{f(M)})^{2} \Phi(r(A))
\end{aligned}
$$

where $r(A)=\min \left\{\frac{A-m I}{M-m}, \frac{M I-A}{M-m}\right\}=\frac{1}{2} I-\frac{1}{M-m}\left|A-\frac{M+m}{2} I\right|$ and

$$
\mu(m, M, f)=\max \left\{\frac{1}{f(t)}\left(\frac{M-t}{M-m} f(m)+\frac{t-m}{M-m} f(M)\right): t \in[m, M]\right\} .
$$


Proof. On account of the assumptions, from parts of (6), we have

$$
\begin{aligned}
f\left(t-(\sqrt{m}-\sqrt{M})^{2} r(t)\right) & \leq f\left(m^{\frac{M-t}{M-m}} M^{\frac{t-m}{M-m}}\right) \\
& \leq L(t)-(\sqrt{f(m)}-\sqrt{f(M)})^{2} r(t)
\end{aligned}
$$

where

$$
L(t)=\frac{M-t}{M-m} f(m)+\frac{t-m}{M-m} f(M) .
$$

Note that inequality (8) holds for all $t \in[m, M]$. On the other hand, $\sigma(A) \subseteq[m, M]$, which, by virtue of monotonicity principle (7) for operator functions, yields the series of inequalities

$$
\begin{aligned}
f\left(A-(\sqrt{m}-\sqrt{M})^{2} r(A)\right) & \leq f\left(m^{\frac{M I-A}{M-m}} M^{\frac{A-m I}{M-m}}\right) \\
& \leq L(A)-(\sqrt{f(m)}-\sqrt{f(M)})^{2} r(A) .
\end{aligned}
$$

It follows from the linearity and the positivity of the map $\Phi$ that

$$
\begin{aligned}
\Phi\left(f\left(A-(\sqrt{m}-\sqrt{M})^{2} r(A)\right)\right) & \leq \Phi\left(f\left(m^{\frac{M I-A}{M-m}} M^{\frac{A-m I}{M-m}}\right)\right) \\
& \leq \Phi(L(A))-(\sqrt{f(m)}-\sqrt{f(M)})^{2} \Phi(r(A)) .
\end{aligned}
$$

Now, by using [5] Corollary 4.12 we get

$$
\begin{aligned}
\Phi\left(f\left(A-(\sqrt{m}-\sqrt{M})^{2} r(A)\right)\right) & \leq \Phi\left(f\left(m^{\frac{M I-A}{M-m}} M^{\frac{A-m I}{M-m}}\right)\right) \\
& \leq \Phi(L(A))-(\sqrt{f(m)}-\sqrt{f(M)})^{2} \Phi(r(A)) \\
& \leq \mu(m, M, f) f(\Phi(A))-(\sqrt{f(m)}-\sqrt{f(M)})^{2} \Phi(r(A)) .
\end{aligned}
$$

This completes the proof.

As discussed extensively in [6] Cahpter 2, for $f(t)=t^{p}$, we have

$$
\begin{aligned}
\mu\left(m, M, t^{p}\right) & =\max \left\{\frac{1}{t^{p}}\left(\frac{M-t}{M-m} m^{p}+\frac{t-m}{M-m} M^{p}\right): t \in[m, M]\right\} \\
& =\frac{\left(m M^{p}-M m^{p}\right)}{(p-1)(M-m)}\left(\frac{p-1}{p} \frac{M^{p}-m^{p}}{m M^{p}-M m^{p}}\right)^{p} .
\end{aligned}
$$

Now, the following fact can be easily deduced from Theorem 1 and Example 1. 
Corollary 1. Let $A \in \mathcal{B}(\mathcal{H})$ be a positive operator with $\sigma(A) \subseteq[m, M]$ for some scalars $m, M$ with $0<m<M$ and $\Phi$ be a normalized positive linear map from $\mathcal{B}(\mathcal{H})$ to $\mathcal{B}(\mathcal{K})$. Then for any $p<0$,

$$
\begin{aligned}
\Phi\left(A^{p}\right) & \leq \Phi\left(\left(A-(\sqrt{m}-\sqrt{M})^{2} r(A)\right)^{p}\right) \\
& \leq \Phi\left(\left(m^{\frac{A-M I}{M-m}} M^{\frac{m I-A}{M-m}}\right)^{p}\right) \\
& \leq K(m, M, p) \Phi(A)^{p}-\left(m^{p / 2}-M^{p / 2}\right)^{2} \Phi(r(A))
\end{aligned}
$$

where

$$
K(m, M, p)=\frac{\left(m M^{p}-M m^{p}\right)}{(p-1)(M-m)}\left(\frac{p-1}{p} \frac{M^{p}-m^{p}}{m M^{p}-M m^{p}}\right)^{p}
$$

In particular,

$$
\begin{aligned}
\Phi\left(A^{-1}\right) & \leq \Phi\left(\left(A-(\sqrt{m}-\sqrt{M})^{2} r(A)\right)^{-1}\right) \\
& \leq \Phi\left(\left(m^{\frac{A-M I}{M-m}} M^{\frac{m I-A}{M-m}}\right)^{-1}\right) \\
& \leq \frac{(M+m)^{2}}{4 M m} \Phi(A)^{-1}-\left(\frac{(\sqrt{M}-\sqrt{m})^{2}}{M m}\right) \Phi(r(A)) .
\end{aligned}
$$

We note that $K(m, M,-1)=\frac{(M+m)^{2}}{4 M m}$ is the original Kantorovich constant.

Theorem 2. Let all the assumptions of Theorem 1 hold. Then

$$
\begin{aligned}
f\left(\Phi(A)-(\sqrt{m}-\sqrt{M})^{2} r(\Phi(A))\right) & \leq f\left(m^{\frac{M I-\Phi(A)}{M-m}} M^{\frac{\Phi(A)-m I}{M-m}}\right) \\
& \leq \mu(m, M, f) \Phi(f(A))-(\sqrt{f(m)}-\sqrt{f(M)})^{2} r(\Phi(A)) .
\end{aligned}
$$

Proof. By applying a standard functional calculus for the operator $\Phi(A)$ such that $m I \leq \Phi(A) \leq M I$, we get from (8)

$$
\begin{aligned}
f\left(\Phi(A)-(\sqrt{m}-\sqrt{M})^{2} r(\Phi(A))\right) & \leq f\left(m^{\frac{M I-\Phi(A)}{M-m}} M^{\frac{\Phi(A)-m I}{M-m}}\right) \\
& \leq \Phi(L(A))-(\sqrt{f(m)}-\sqrt{f(M)})^{2} r(\Phi(A)) .
\end{aligned}
$$


We thus have

$$
\begin{aligned}
f\left(\Phi(A)-(\sqrt{m}-\sqrt{M})^{2} r(\Phi(A))\right) & \leq f\left(m^{\frac{M I-\Phi(A)}{M-m}} M^{\frac{\Phi(A)-m I}{M-m}}\right) \\
& \leq L(\Phi(A))-(\sqrt{f(m)}-\sqrt{f(M)})^{2} r(\Phi(A)) \\
& =\Phi(L(A))-(\sqrt{f(m)}-\sqrt{f(M)})^{2} r(\Phi(A)) \\
& \leq \mu(m, M, f) \Phi(f(A))-(\sqrt{f(m)}-\sqrt{f(M)})^{2} r(\Phi(A))
\end{aligned}
$$

where at the last step we used the basic inequality [5] Corollary 4.12.

Hence, the proof is complete.

As a corollary of Theorem 2 we have:

Corollary 2. Let all the assumptions of Corollary 1 hold. Then for any $p<0$

$$
\begin{aligned}
\Phi(A)^{p} & \leq\left(\Phi(A)-(\sqrt{m}-\sqrt{M})^{2} r(\Phi(A))\right)^{p} \\
& \leq\left(m^{\frac{M I-\Phi(A)}{M-m}} M^{\frac{\Phi(A)-m I}{M-m}}\right)^{p} \\
& \leq K(m, M, p) \Phi\left(A^{p}\right)-\left(\sqrt{m^{p}}-\sqrt{M^{p}}\right)^{2} r(\Phi(A)) .
\end{aligned}
$$

Remark 1. Notice that the inequalities in Corollary 2 are stronger than the inequalities obtained in [11] Corollary 2.1.

Recall that if $f$ is operator convex, the solidarities [12] or the perspective [13] of $f$ is defined by

$$
\mathcal{P}_{f}(A \mid B)=A^{\frac{1}{2}} f\left(A^{-\frac{1}{2}} B A^{-\frac{1}{2}}\right) A^{\frac{1}{2}} .
$$

Using a series of inequalities (6) we have the upper bounds of the perspective for non-negative decreasing geometrically convex function (not necessary operator convex $f$ ). We use the same symbol $\mathcal{P}_{f}(A \mid B)$ for a simplicity.

Proposition 1. Let $A, B>0$ with $m A \leq B \leq M A$ for some scalars $0<m<M$. For a non-negative decreasing geometrically convex function $f$, we have

$$
\begin{aligned}
\mathcal{P}_{f}(A \mid B) & \leq A^{1 / 2} f\left(A^{-1 / 2} B A^{-1 / 2}-(\sqrt{m}-\sqrt{M})^{2} r(A, B)\right) A^{1 / 2} \\
& \leq A^{1 / 2} f\left(m^{\frac{M I-A^{-1 / 2} B A^{-1 / 2}}{M-m}} M^{\frac{A^{-1 / 2} B A^{-1 / 2}-m I}{M-m}}\right) A^{1 / 2} \\
& \leq A^{1 / 2} f(m)^{\frac{M I-A^{-1 / 2} B A^{-1 / 2}}{M-m}} f(M)^{\frac{A^{-1 / 2} B A^{-1 / 2}-m I}{M-m}} A^{1 / 2} \\
& \leq \frac{M f(m)-m f(M)}{M-m} A+\frac{f(M)-f(m)}{M-m} B-(\sqrt{f(m)}-\sqrt{f(M)})^{2} A^{1 / 2} r(A, B) A^{1 / 2} \\
& \leq \frac{M f(m)-m f(M)}{M-m} A+\frac{f(M)-f(m)}{M-m} B,
\end{aligned}
$$


where

$$
\begin{aligned}
r(A, B) & =\min \left\{\frac{A^{-1 / 2} B A^{-1 / 2}-m I}{M-m}, \frac{M I-A^{-1 / 2} B A^{-1 / 2}}{M-m}\right\} \\
& =\frac{1}{2} I-\frac{1}{M-m}\left|A^{-1 / 2} B A^{-1 / 2}-\frac{M+m}{2} I\right| .
\end{aligned}
$$

Author Contributions: The work presented here was carried out in collaboration between all authors. The study was initiated by the first author. The first author played also the role of the corresponding author. All authors contributed equally and significantly in writing this article. All authors have read and approved the final manuscript.

Funding: This research was funded by JSPS KAKENHI Grant Number 16K05257.

Acknowledgments: The authors would like to express their hearty thanks to the referees for their valuable comments.

Conflicts of Interest: The authors declare no conflict of interest.

\section{References}

1. Marshall, A.W.; Olkin, I. Matrix versions of Cauchy and Kantorovich inequalities. Aequ. Math. 1990, 40, 89-93. [CrossRef]

2. Kantorovich, L.V. Functional analysis and applied mathematics. Uspehi Matem. Nauk 1948, 3, 89-185. (In Russian)

3. Bourin, J.C. Matrix versions of some classical inequalities. Linear Algebra Appl. 2006, 416, 890-907. [CrossRef]

4. Fujii, M.; Zuo, H.; Cheng, N. Generalization on Kantorovich inequality. J. Math. Inequal. 2013, 7, 517-522. [CrossRef]

5. Mićić, J.; Carić, J.P.; Seo, Y.; Tominaga, M. Inequalities for positive linear maps on Hermitian matrices. J. Math. Inequal. Appl. 2000, 3, 559-591.

6. Furuta, T.; Mićić, J.; Carić, J.P.; Seo, Y. Mond-Pečarić Method in Operator Inequalities; Element: Guernsey, France, 2005.

7. Moradi, H.R.; Gümüş, I.H.; Heydarbeygi, Z. A glimpse at the operator Kantorovich inequality. Linear Multilinear Algebra 2018. [CrossRef]

8. Montel, P. Sur les functions convexes et les fonctions sousharmoniques. J. Math. 1928, 9, 29-60.

9. Bourin, J.C.; Hiai, F. Jensen and Minkowski inequalities for operator means and anti-norms. Linear Algebra Appl. 2014, 456, 22-53. [CrossRef]

10. Kittaneh, F.; Manasrah, Y. Improved Young and Heinz inequalities for matrices. J. Math. Anal. Appl. 2010, 361, 262-269. [CrossRef]

11. Sababheh, M.; Moradi, H.R.; Furuichi, S. Exponential inequalities for positive linear mappings. J. Funct. Spaces 2018, 2018, 5467413. [CrossRef]

12. Fujii, J.I.; Fujii, M.; Seo, Y. An extension of the Kubo-Ando theory: Soridarities. Math. Japonica 1990, 35, 387-396.

13. Ebadian, A.; Nikoufar, I.; Gordji, M.E. Perspectives of matrix convex functions. Proc. Natl. Acad. Sci. USA 2011, 108, 7313-7314. [CrossRef]

(C) 2019 by the authors. Licensee MDPI, Basel, Switzerland. This article is an open access article distributed under the terms and conditions of the Creative Commons Attribution (CC BY) license (http://creativecommons.org/licenses/by/4.0/). 